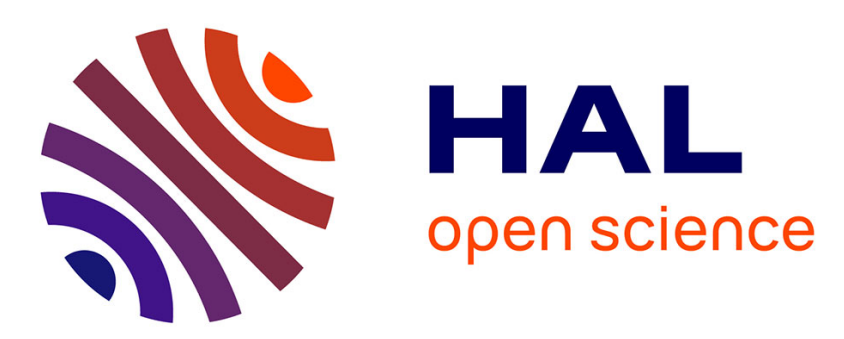

\title{
ASYMMETRIC PATCH SIZE DISTRIBUTION LEADS TO DISRUPTIVE SELECTION ON DISPERSAL
}

François Massol, Anne Duputié, Patrice David, Philippe Jarne

\section{- To cite this version:}

François Massol, Anne Duputié, Patrice David, Philippe Jarne. ASYMMETRIC PATCH SIZE DISTRIBUTION LEADS TO DISRUPTIVE SELECTION ON DISPERSAL. Evolution - International Journal of Organic Evolution, 2010, 65 (2), pp.490-500. 10.1111/j.1558-5646.2010.01143.x . hal02356719

\section{HAL Id: hal-02356719 \\ https://hal.science/hal-02356719}

Submitted on 8 Nov 2019

HAL is a multi-disciplinary open access archive for the deposit and dissemination of scientific research documents, whether they are published or not. The documents may come from teaching and research institutions in France or abroad, or from public or private research centers.
L'archive ouverte pluridisciplinaire HAL, est destinée au dépôt et à la diffusion de documents scientifiques de niveau recherche, publiés ou non, émanant des établissements d'enseignement et de recherche français ou étrangers, des laboratoires publics ou privés. 


\title{
ASYMMETRIC PATCH SIZE DISTRIBUTION LEADS TO DISRUPTIVE SELECTION ON DISPERSAL
}

\author{
François Massol ${ }^{1,2,3,4,5}$, Anne Duputié ${ }^{1,2,6}$, Patrice David ${ }^{1,7}$, and Philippe Jarne ${ }^{1,8}$ \\ ${ }^{1}$ Centre d'Écologie Fonctionnelle et Évolutive - UMR 5175, campus CNRS, 1919, route de Mende, 34293 Montpellier \\ Cedex 5, France \\ ${ }^{2}$ University of Texas at Austin, Section of Integrative Biology, Austin, TX 78712 \\ ${ }^{3}$ CEMAGREF - UR HYAX, 3275, route de Cézanne - Le Tholonet, CS 40061, 13182 Aix-en-Provence Cedex 5, France \\ ${ }^{4}$ Centre Alpin de Recherche sur les Réseaux Trophiques des Écosystèmes Limniques (INRA), 75, avenue de Corzent-BP 511, \\ 74203 Thonon-les-Bains Cedex, France \\ ${ }^{5}$ E-mail: francois.massol@cemagref.fr \\ ${ }^{6}$ E-mail: anne.duputie@ens-lyon.org \\ ${ }^{7}$ E-mail: patrice.david@cefe.cnrs.fr \\ ${ }^{8}$ E-mail: philippe.jarne@cefe.cnrs.fr
}

Received July 29, 2009

Accepted February 16, 2010

\begin{abstract}
Numerous models have been designed to understand how dispersal ability evolves when organisms live in a fragmented landscape. Most of them predict a single dispersal rate at evolutionary equilibrium, and when diversification of dispersal rates has been predicted, it occurs as a response to perturbation or environmental fluctuation regimes. Yet abundant variation in dispersal ability is observed in natural populations and communities, even in relatively stable environments. We show that this diversification can operate in a simple island model without temporal variability: disruptive selection on dispersal occurs when the environment consists of many small and few large patches, a common feature in natural spatial systems. This heterogeneity in patch size results in a high variability in the number of related patch mates by individual, which, in turn, triggers disruptive selection through a high per capita variance of inclusive fitness. Our study provides a likely, parsimonious and testable explanation for the diversity of dispersal rates encountered in nature. It also suggests that biological conservation policies aiming at preserving ecological communities should strive to keep the distribution of patch size sufficiently asymmetric and variable.
\end{abstract}

KEY WORDS: Adaptive dynamics, dispersal evolution, evolutionary branching, evolutionarily stable strategy, habitat heterogeneity, kin selection.

Many species have patchy spatial distributions, that is, form metapopulations (Hanski and Gilpin 1997), consisting of a set of discrete populations connected by dispersal. A central theme in metapopulation biology is the evolution of dispersal (Gandon and Michalakis 2001; Levin et al. 2003; Ronce 2007). The purpose of studies on this theme is twofold (Ronce 2007): first, to describe how different factors such as extrinsic perturbations (Comins et al.
1980; Ronce et al. 2000; Gandon and Michalakis 2001; Parvinen 2002; Jansen and Vitalis 2007), habitat heterogeneity (Balkau and Feldman 1973; Roff 1975; Hastings 1983; McPeek and Holt 1992; Mathias et al. 2001), inbreeding (Bengtsson 1978; Roze and Rousset 2005), the costs and risks associated with dispersing (Hamilton and May 1977; Comins et al. 1980; Ronce et al. 2000; Gandon and Michalakis 2001), or competition with related 
individuals (van Valen 1971; Hamilton and May 1977; Gandon and Michalakis 2001; Cadet et al. 2003), affect the evolutionarily stable strategy (ESS) for dispersal; second, to assess which conditions lead to disruptive selection and the emergence of a polymorphism for dispersal (Holt and McPeek 1996; Doebeli and Ruxton 1997; Mathias et al. 2001; Parvinen 2002).

Although most simple models predict the existence of an ESS for dispersal (e.g., Hamilton and May 1977; Comins et al. 1980; Cadet et al. 2003), many empirical observations report high variability in dispersal strategies within species or among closely related species (Roff 1986; Hazell et al. 2005; Hanski and Saccheri 2006). This suggests that additional factors are needed to explain dispersal polymorphisms. For instance, environmental variability (Roff 1975; McPeek and Holt 1992; Mathias et al. 2001), fluctuating population dynamics (Holt and McPeek 1996; Doebeli and Ruxton 1997), or random catastrophes in heterogeneous habitats (Parvinen 2002) may lead to disruptive selection on dispersal rates when habitat patches are spatially heterogeneous (Kisdi 2002). Yet, even in relatively stable environments such as coral reefs (Wood 2001), tropical rainforests (Primack and Corlett 2005), or sub-arctic meadows (Hanski and Saccheri 2006), there is evidence for both intra and interspecific variation in dispersal rates. To date, theoretical results do not explain the evolutionary diversification of dispersal rates in stable environments (Hastings 1983; Ronce 2007).

Field ecologists often observe metapopulations that are likely to exhibit an important heterogeneity in carrying capacity among patches. Indeed, carrying capacity, as estimated from simple proxies (e.g., pond perimeters for freshwater mollusks, meadow patch areas for some butterflies) always show some, often conspicuous, variability in natural systems (Hanski and Gilpin 1997; Clobert et al. 2001; Brock et al. 2008). Intuitively, when carrying capacities are uniformly low, high-dispersal types (drifters) overpower low-dispersal types (dwellers) because offspring remaining in their natal patch tend to compete with related patch mates (Hamilton and May 1977). Conversely, when carrying capacities are high, dwellers dominate because dispersal is costly and kin competition is weak. More generally, the dispersal ESS is determined by the balance between kin competition and dispersal cost (Gandon and Michalakis 2001). An open question is what happens when carrying capacities are variable among populations.

In this study, we propose a model based solely on dispersal cost and kin competition in which the diversification of dispersal rates results from heterogeneous population sizes.

\section{Methods MODEL BASICS}

We considered a metapopulation (a set of populations connected by dispersal) consisting of an infinite number of patches, with a specified distribution of carrying capacities. As an approximation, patches were considered to have fixed population sizes (carrying capacities, $K$, with frequencies $\pi_{K}$ ), that is, resources freed by an individual's death were immediately allocated to a newly settled individual, and there was no catastrophe wiping out a whole population. The model obeyed the following rules (Fig. 1): (1) all individuals had the same mortality rate; (2) a dead individual was immediately replaced by either a resident or immigrant (dispersed) offspring; (3) all individuals had the same fecundity; (4) individuals of strategy $d$ sent a proportion $d$ of their offspring to the propagule pool ( $1-d$ remained in their natal patch); (5) a proportion $c$ of propagules (the dispersal cost) died before reaching a randomly chosen destination patch. Following these rules, the probability that the next death (in a patch with carrying capacity $K$ ) affected a type $s$ individual (local abundance $k_{s}$ ) was $k_{s} / K$, and the probability that a dead individual was replaced by a type $s$ individual (average abundance over all patches $\bar{k}_{s}$ ) was proportional to $\left(1-d_{s}\right) k_{s}+(1-c) d_{s} \bar{k}_{s}$. When dispersal is monomorphic, our model was equivalent to Hubbell's neutral community model with no speciation (Hubbell 2001).

Based on these five elementary rules, we studied the effects of selection by testing whether a rare mutant with dispersal strategy $d$ increased in frequency in a resident metapopulation with strategy $\hat{d}$. In a homogeneous population, this is usually assessed by computing the reproductive rate of the mutant (Charlesworth $1994)$; in a metapopulation, the relevant quantity $\left(R_{m}\right)$ is the average number of dispersers produced by a population founded by a single mutant disperser (Chesson 1984; Metz and Gyllenberg 2001; Massol et al. 2009). Assuming that mutations are rare and have small individual effects, the adaptive dynamics method (Hofbauer and Sigmund 1990; Geritz et al. 1998; Champagnat et al. 2006) can be used to determine whether natural selection is stabilizing (ESS) or disruptive (branching point), provided singular strategies are convergence stable (CS, Geritz et al. 1998).

\section{INVASIBILITY ANALYSIS}

When type 1 was very rare, it could invade a metapopulation entirely occupied by type 2 only if $R_{m}\left(d_{1}, d_{2}\right)>1$, where $R_{m}\left(d_{l}\right.$, $d_{2}$ ) was defined as the mean number of dispersers produced by a type 1 colony between its foundation and its eventual demise, in the absence of type 1 immigrants (Chesson 1984; Metz and Gyllenberg 2001; Massol et al. 2009). The computation of $R_{m}$ yielded (Appendix S1):

$$
\begin{aligned}
R_{m}\left(d_{1}, d_{2}\right)= & \sum_{K}\left[\frac{(1-c) d_{1} K \pi_{K}}{\left(1-d_{2}\right)(K-1)+(1-c) d_{2} \bar{K}}\right] \\
& { }_{2} F_{1}\left[1,1-K ; 2-K-\frac{(1-c) d_{2} \bar{K}}{1-d_{2}} ; \frac{1-d_{1}}{1-d_{2}}\right],
\end{aligned}
$$



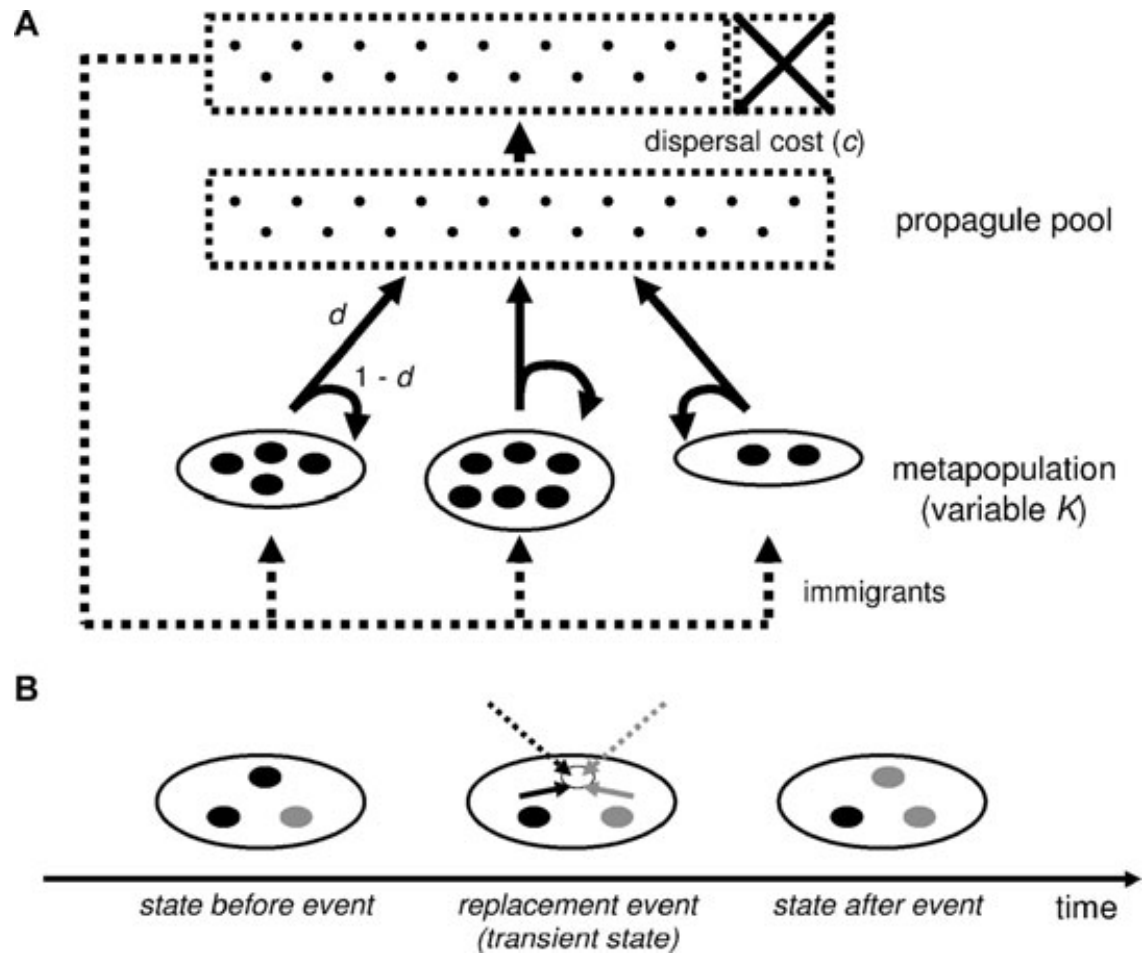

Figure 1. Structure of the metapopulation model used to study the evolution of dispersal. (A) Functioning of the metapopulation. Open ovals indicate patches, with variable carrying capacity $(K)$, closed ovals are individuals. The dotted box represents the propagule pool, before and after applying the cost of dispersal, which removes a fraction c of propagules. Solid arrows represent the flows of offspring (a proportion $d$ is dispersed, and $1-d$ remains in the natal patch); dotted arrows, the flows of propagules (dispersed offspring). (B) Competition between different types. Two types (black and gray, that differ with respect to their dispersal rates) compete in a fully occupied patch containing three individuals. A black individual dies; a stochastic competition process takes place immediately among resident offspring (solid arrows) and immigrant propagules (dotted arrows) from both types. In our example, the empty site is eventually occupied by a gray individual.

where ${ }_{2} F_{1}[a, b ; c ; z]$ is the hypergeometric series. Singular strategies of dispersal were found by nullifying the selection gradient, that is, $d$ was a singular strategy when $\left[\partial R_{m} / \partial d_{1}\right]_{d_{1}=d_{2}=d}=0$ (Hofbauer and Sigmund 1990). In turn, a singular strategy was CS when $\left[\partial^{2} R_{m} / \partial d_{1}^{2}\right]_{d_{1}=d_{2}=d}<\left[\partial^{2} R_{m} / \partial d_{2}^{2}\right]_{d_{1}=d_{2}=d}$ and an ESS when $\left[\partial^{2} R_{m} / \partial d_{1}^{2}\right]_{d_{1}=d_{2}=d}=0$ (Geritz et al. 1998).

\section{SIMULATIONS}

To confirm analytical predictions, we performed simulations of evolutionary processes in metapopulations. We simulated metapopulations in a landscape containing $N=1000$ patches. The carrying capacities of the patches followed a truncated geometric distribution of parameter $p$, that is, $\pi_{K}=p(1-p)^{K-1}$ for $K<K_{\max }$ and $\pi_{K_{\max }}=(1-p)^{K_{\max }-1}$. The difference between the truncated and true geometric distributions was small as long as $K_{\max }$ was high enough. We took $K_{\max } \approx-\log (N) / \log (1-p)$, so that the probability that a patch had more than $K_{\max }$ microsites was $\approx 1 / N$ under the corresponding true geometric distribution.

A simulation started with the generation of the landscape. All patches were then filled with a unique type that had a dispersal strategy of 0.5 (this has no consequence on the result of the sim- ulations). At each simulation step, one microsite was randomly chosen among all microsites from all patches, its occupant was killed and there was a lottery competition for replacement in that microsite (according to the five model rules). Once the type of the replacing individual was found, there was a 0.001 probability that it was in fact a mutant that deviated from its ancestral phenotype (this implies that dispersal is maternally controlled). The dispersal strategy of a mutant was computed as the dispersal strategy of its ancestral type plus a random Gaussian variable (standard deviation $=0.1$ ). As dispersal cannot be higher than 1 or lower than 0 , the difference between the ancestral and mutant dispersal strategies was halved when the latter was off limit. If this did not suffice to obtain a suitable mutant phenotype, the mutant dispersal strategy was equal to its ancestral strategy.

Each simulation ran for $80,000,000$ steps (even very large metapopulations, i.e., with low $p$ had evolutionarily converged before the 40,000,001st step). Every 200,000 steps, the metapopulation state was summarized (types were grouped by dispersal strategies in 101 equally spaced classes, from $d=0.00$ to $d=1.00$ ) and recorded. Thus, at the end of a simulation, we obtained a historical record of 400 metapopulation states (e.g., Fig. S1 and S2). 
The steady state of the metapopulation was taken as the average of the last 200 records (i.e., the average of recorded metapopulation states between the $40,000,001$ st and $80,000,000$ th steps). Because disruptive selection occurred for $c<p<(1+c) / 2$ (see Appendix S2), we took $c=0.33$ for all simulations (so that the $p$ scale could be divided in three equal parts) and varied parameter $p$ in the interval $[0.05 ; 0.95]$ in steps of size 0.01 .

\section{ROBUSTNESS TO DEMOGRAPHIC STOCHASTICITY}

The simulations conducted above assumed fixed population sizes, and therefore no demographic stochasticity. To assess whether our results were robust to relaxing this assumption, we also ran simulations in a metapopulation in which the ratio of birth rate to death rate ( $b / m$ ratio) was finite (i.e., replacements of dead individuals were not immediate). In this model, local population numbers were subject to stochastic fluctuations because an empty site within a patch could remain so while other individuals died or were recruited in other sites. We ran simulations in a landscape of patches following a truncated geometric distribution of patch carrying capacity (as above), with $c=0.33$ and $b / m=100$. Details on the model with demographic stochasticity can be found in Appendix S3.

\section{FIELD DATA ON PATCH SIZE DISTRIBUTIONS}

To confront model predictions with data, we computed the distribution of patch size in seven landscapes representing very different situations: pond sizes in the Grande Terre of Guadeloupe island (West Indies), which are home to several species of freshwater mollusks (P. David et al., unpubl. data); the populations of large cities in People's Republic of China (United Nations' data for year 2003), which suit our model from the viewpoint of human parasites; the areas of dry meadow patches on Aland islands, harboring populations of the Glanville fritillary butterfly Melitaea cinxia (I. Hanski, pers. comm.); the areas of islands in the Tuamotu archipelago (French Polynesia, data from the "Institut de la statistique de Polynésie française"); the size of forest patches in Pennsylvania (USA, US geological survey data); the areas of Svalbard islands (Norway); and the areas of coral reefs in the Northern Florida Keys (USA, J. C. Brock and M. PalaseanuLovejoy, pers. comm.). For each dataset, we assessed whether disruptive selection on dispersal was likely for organisms that can inhabit these habitat patches.

\section{Results}

\section{ANALYTICAL PREDICTIONS}

In our model, the evolution of dispersal is affected by the cost of dispersal $(c)$, and three moments of the distribution of patch carrying capacities: its mean $(\bar{K}=\mathrm{E}[K])$, squared coefficient of variation $\left(\gamma_{2}=\mathrm{E}\left[(K-\bar{K})^{2}\right] / \bar{K}^{2}\right)$, and standardized skewness $\left(\gamma_{3}=\mathrm{E}\left[(K-\bar{K})^{3}\right] /\left(\bar{K}^{3} \gamma_{2}^{3 / 2}\right)\right)$. From equation (1), the selection gradient is given by

$$
\left[\frac{\partial R_{m}}{\partial d_{1}}\right]_{d_{1}=d_{2}=d}=\frac{1-\left(c+\gamma_{2}\right) \bar{K} d}{d(1-d+d(1-c) \bar{K})}
$$

which yields the unique singular strategy, $d^{*}$ :

$$
d^{*}=\operatorname{Min}\left[\frac{1}{\left(c+\gamma_{2}\right) \bar{K}}, 1 .\right]
$$

The ESS conditions are obtained through double differentiation of equation (1). Following these conditions (Appendix S1), the evolution of dispersal leads to a unique ESS either when

$$
\left(c+\gamma_{2}\right) \bar{K}<1
$$

or

$$
\gamma_{3}<2 \gamma_{2}^{1 / 2}+\frac{(c \bar{K}-1)}{\bar{K}} \gamma_{2}^{-1 / 2}+\frac{c(\bar{K}-1)}{\bar{K}} \gamma_{2}^{-3 / 2} \text {. }
$$

When inequality (4) is satisfied, evolutionary stability is always achieved because $d^{*}=1$ is not an interior extremum of the fitness function, but rather results from a trait constraint and directional selection (eq. 3). When $\left(c+\gamma_{2}\right) \bar{K}>1$ and inequality (5) is satisfied, $d^{*}$ is a maximum of $R_{m}\left(d, d^{*}\right)$, and thus is an ESS.

Stabilizing selection may occur for any value of $\gamma_{2}$, but only for a narrow range of $\gamma_{3}$ values (Fig. 2). Under conditions of stabilizing selection, an increase in the cost of dispersal $(c)$, in the average carrying capacity $(\bar{K})$, or in the heterogeneity of carrying capacities $\left(\gamma_{2}\right)$ selects against dispersal (eq. 3). In other words, three separate processes select against dispersal: (1) the direct cost of dispersal $(c)$; (2) the indirect cost due to the asymmetry of migrant flow between small and large patches (Holt 1985), embodied in the factor $\gamma_{2}$; and (3) the fact that individuals are less related to each other in a metapopulation with high $\bar{K}$.

When the distribution of carrying capacity is sufficiently variable and positively skewed (large $\gamma_{2}$ and $\gamma_{3}$ ), so that neither inequality (4) nor (5) is satisfied, disruptive selection occurs, and two or more strategies emerge. Disruptive selection is more likely when the dispersal cost is low (inequality [5]), and the mean carrying capacity is intermediate (just above $1 /\left(\gamma_{2}+c\right)$; inequalities [4] and [5]). Although inequality (4) is simple to understand (if inequality [4] is satisfied, $d^{*}=1$ and thus selection is directional at the singular strategy, precluding disruptive selection), inequality (5) needs a little re-writing to be clearly interpreted. Noting $E_{\text {exp }}$ and $\operatorname{Var}_{\text {exp }}$, the operators for mean and variance of metapopulation quantities experienced by individuals, and $W_{\mathrm{IF}}(K)$ the inclusive fitness (sensu Ajar 2003) of individuals in $K$-patches, inequality (5) is equivalent to (Appendix S1)

$$
d^{*} \mathrm{E}_{\exp }[K-1] \operatorname{Var}_{\exp }\left[W_{I F}(K)\right]<1 .
$$




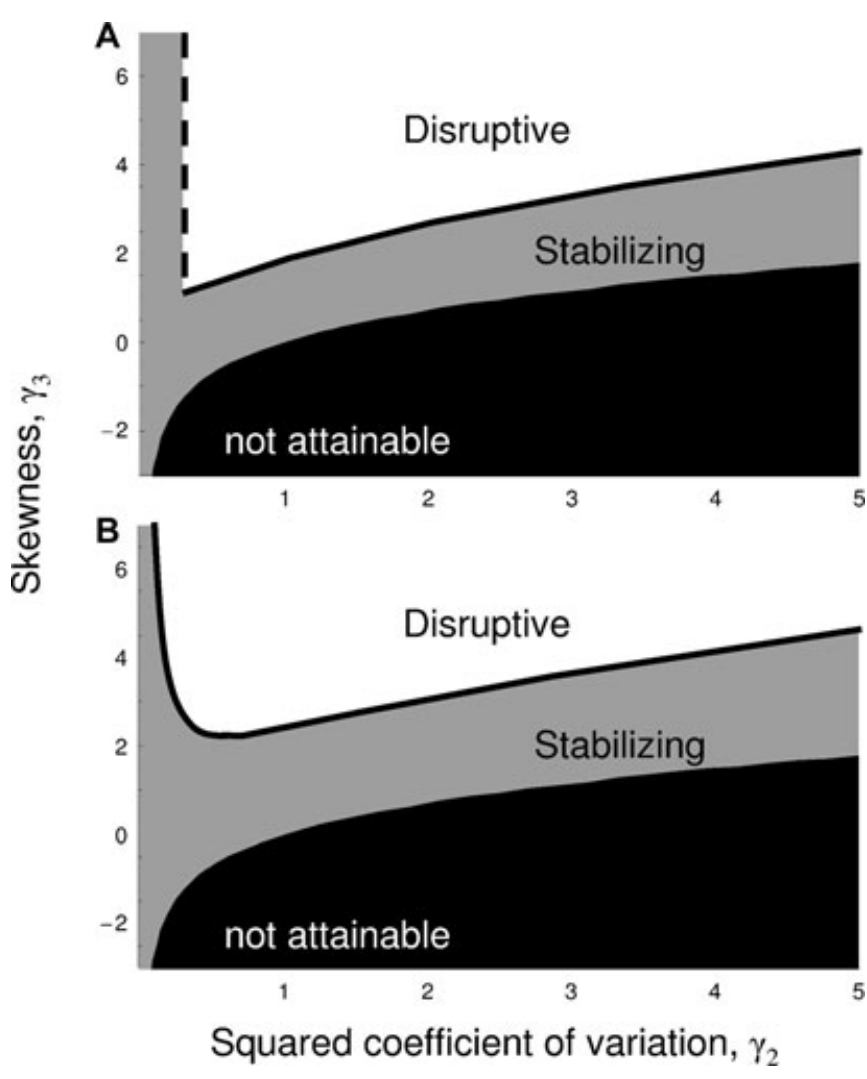

Figure 2. Conditions for disruptive or stabilizing selection on dispersal. Abscissas indicate the value of the coefficient of variation of carrying capacities $\left(\gamma_{2}\right)$, ordinates represent the value of the skewness of carrying capacities $\left(\gamma_{3}\right)$. The black region is mathematically impossible because there is a lower bound $(=1)$ to the value of the carrying capacity of a patch. In the gray region, there is a unique ESS for the dispersal level (stabilizing selection). In the white region, selection on dispersal is disruptive and adaptive dynamics display an evolutionary branching point. Thick lines indicate the first (dashed line) and second (solid line) boundaries separating domains of disruptive and stabilizing selection, corresponding to inequalities (4) and (5), respectively. The cost of dispersal is fixed at $\boldsymbol{c}=\mathbf{0 . 2}$, and the mean carrying capacity is either (A) $\bar{K}=2$ or (B) $\bar{K}=\infty$.

Inequality (6) means that disruptive selection only occurs when individuals experience sufficiently variable inclusive fitness among patch types $\left(\operatorname{Var}_{\exp }\left[W_{I F}(K)\right]\right)$, and it is favored when the average number of intrapatch neighbors $\left(\mathrm{E}_{\exp }[K-1]\right)$ and the singular dispersal strategy $\left(d^{*}\right)$ are high. The variance in inclusive fitness in inequality (6) is the term that increases with the skewness of the distribution of carrying capacity (Appendix S1), i.e., selective pressures on dispersal are more variable in a metapopulation with a more skewed distribution of carrying capacity, and this in turn allows for disruptive selection on dispersal to take place.

Inequalities (4) and (5) implicitly define two parameter boundaries separating stabilizing and disruptive selection regimes (Fig. 2). The first boundary (inequality [4]) is defined by a mini- mum value on carrying capacity variability $\gamma_{2}$ (equal to $1 / \bar{K}-c$ ) under which $d^{*}=1$ is selected. The second boundary (inequality [5]) is defined by a minimum value on carrying capacity skewness under which selection is stabilizing. When $c \bar{K}>1$, only the second boundary (inequality [5]) exists (Fig. 2B).

Under a disruptive selection regime close to either parameter boundary (as defined by inequalities [4] and [5]), two strategies (called drifters and dwellers, for high- and low-dispersal types, respectively) emerge from the branching point and stably coexist. Dwellers disappear when carrying capacity variability becomes too low (i.e., when crossing the first boundary; inequality [4]) because selection becomes directional, whereas drifters do not emerge when carrying capacity distribution is less skewed (i.e., when crossing the second boundary; inequality [5]) because inclusive fitness is not variable enough, which precludes disruptive selection to occur (inequality [6]). Stating that drifters disappear at the second boundary may be deemed arbitrary. However, inequality (6), which also defines the second boundary, implies an upper bound for $d^{*}$ under stabilizing selection: $d^{*}<1 / \mathrm{E}_{\exp }$ $[K-1] \operatorname{Var}_{\exp }\left[W_{I F}(K)\right]$, hence our choice of writing that drifters disappear at this boundary.

\section{SIMULATION RESULTS}

Analytical predictions are corroborated by simulations of finite metapopulations (Fig. 3). As an example, when carrying capacities follow a geometric distribution of parameter $p$ (which controls both the variance and skewness of the distribution), our model predicts disruptive selection on dispersal when $c \leq p \leq(1+c) / 2$, and stabilizing selection otherwise (Appendix S2). This is indeed what we observe in simulations (Fig. 3): selection is stabilizing at low and high values of $p$, and the observed dispersal level agrees with analytical predictions (eq. 3; see Fig. 3A and S1). When $p$ is lower than $c$, drifters disappear, whereas dwellers do when $p>(1+c) / 2$ (Fig. 3). For intermediate values of $p$, we observe evolutionary branching, so that the variance of dispersal level is increased (Fig. 3B): drifters and dwellers coexist (Fig. 3A and S2).

\section{THE EFFECT OF DEMOGRAPHIC STOCHASTICITY}

When population size is allowed to vary stochastically, simulations of finite metapopulations corroborate predictions made under the model with fixed population size (Fig. 3C, D). The observed values for dispersal agree with the ESS prediction when $p<c$ (Fig. 3C). Disruptive and stabilizing selection regimes occur almost as predicted under the simple model with fixed population sizes (Fig. 3D). The only barely noticeable differences between the two series of simulations happen in the "disruptive selection" range: when demographic stochasticity is added, drifters tend to have a lower dispersal rate (and to be less abundant, results 

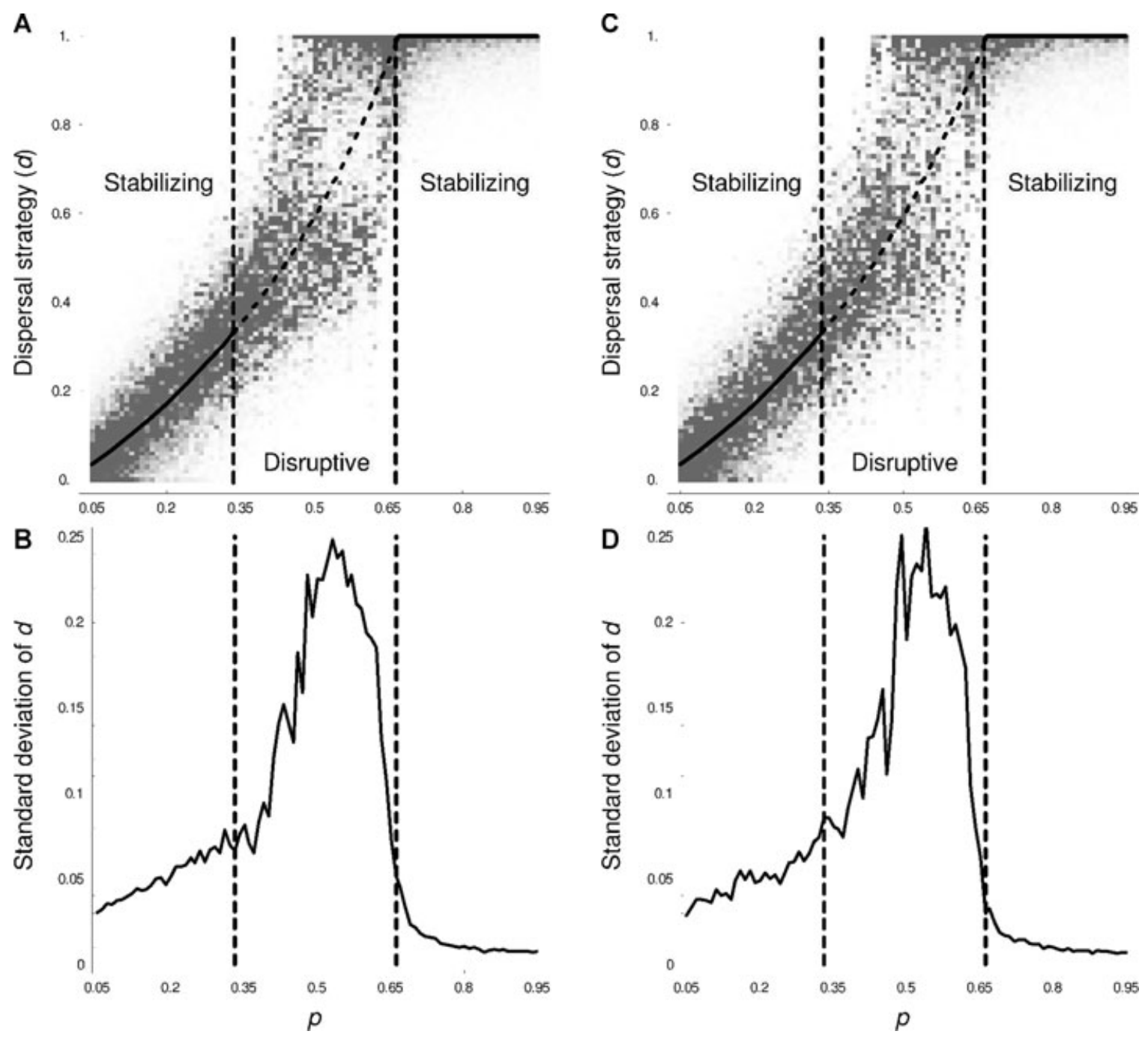

Figure 3. Simulations of metapopulations with geometric distribution of carrying capacities. (A, C) Distribution of dispersal types ( $d$, ordinates) for different values of the geometric distribution parameter ( $p$, abscissas). Darker gray shades indicate a higher frequency of the corresponding dispersal type. The thick black line indicates the theoretical value of the ESS (continuous line) or branching point (dashed line). (B, D) Standard deviation of dispersal distributions (ordinates) for different values of $p$ (abscissas). In all panels, vertical dashed lines indicate the theoretical limits separating stabilizing and disruptive selection on dispersal (Appendix S2). The cost of dispersal is $c=$ 0.33. Panels (A) and (B) correspond to the model with fixed population sizes; panels (C) and (D), to the general model with demographic stochasticity.

not shown) than when population sizes are constant (Fig. 3A, C). However, this effect does not contradict our prediction on the occurrence of disruptive selection under predicted parameter values.

\section{APPLICATION TO FIELD DATA}

An interesting property of our model is that it provides a sufficient criterion for the occurrence of disruptive selection on dispersal (this criterion is based on the worst possible conditions for disruptive selection to occur; see Appendix S1). When $\bar{K}$ is large, selection is disruptive for $c<c_{\max }$, with $c_{\max }=\left(\gamma_{3}-\right.$ $\left.2 \gamma_{2}^{1 / 2}\right) \gamma_{2}^{3 / 2} /\left(1+\gamma_{2}\right)$. Hence, when $c_{\max }>1$, the distribution of carrying capacity always favors disruptive selection on dispersal. This is interesting from an empirical point of view because it is often difficult to measure carrying capacities in natural metapopulations. Indeed, this requires counting individuals in each population, which is unfeasible with most small-sized organisms. Moreover, actual dispersal costs are also difficult to measure. For these two reasons, we cannot directly predict whether stabilizing or disruptive selection would occur in organisms that have not been thoroughly studied. However, $c_{\max }$ can be used to evaluate whether disruptive selection on dispersal due to patch size heterogeneity is likely when simple proxies of carrying capacities, such as patch area, are available. 
Table 1. Distributions of carrying capacity proxies in real datasets.

\begin{tabular}{llcrrr}
\hline Dataset & Proxy (unit) & No. of patches & $\gamma_{2}$ & $\gamma_{3}$ & $c_{\max }$ \\
\hline Ponds (Guadeloupe) $^{1}$ & width (m) & 274 & 1.7 & 4.5 & 1.5 \\
Population in big cities (China) $^{2}$ & pop. size (inhab.) & 664 & 1.5 & 6.7 & 3.2 \\
Dry meadows (Åland islands, Finland) $^{3}$ & area (m²) & 4,109 & 7.3 & 11.1 & 13.5 \\
Tuamotu archipelago (French Polynesia) $^{4}$ & area $\left(\mathrm{km}^{2}\right)$ & 118 & 10.7 & 8.1 & 4.7 \\
Forest patches (Pennsylvania, USA) $^{5}$ & area $\left(\mathrm{m}^{2}\right)$ & 252 & 44.7 & 12.0 & -8.7 \\
Svalbard islands (Norway) $^{6}$ & area $\left(\mathrm{km}^{2}\right)$ & 11 & 4.5 & 2.7 & -2.7 \\
Coral reefs (Northern Florida Keys, USA) $^{7}$ & area $\left(\mathrm{m}^{2}\right)$ & 1,034 & 1.3 & 3.8 & 1.0 \\
\hline
\end{tabular}

\footnotetext{
${ }^{1}$ P. David et al., unpubl. data.

${ }^{2}$ Data from http://unstats.un.org/unsd/demographic/products/dyb/DYB2003/Table08.pdf accessed on September 16th, 2007.

${ }^{3}$ I. Hanski, pers. comm.; see also Hanski and Saccheri (2006).

${ }^{4}$ Institut de la statistique de Polynésie française.

${ }^{5}$ National Landcover Pattern Database, US geological survey (http://www.Isc.usgs.gov/gis/nlpd/asp/pages/getstate.asp?abbrev=PA).

${ }^{6}$ data obtained http://en.wikipedia.org/wiki/List_of_islands_of_Norway_by_area.

7J. C. Brock and M. Palaseanu-Lovejoy, pers. comm.; see also Brock et al. (2008).
}

The seven patch size distributions that we collected are positively skewed (Table 1). $c_{\max }$ largely exceeds 1 in all but two datasets (forest patches in Pennsylvania and Svalbard islands) and is almost equal to 1 in Northern Florida Keys coral reef patches. This suggests that disruptive selection on dispersal can operate under widely different natural settings.

\section{Discussion \\ THE SKEWNESS OF PATCH SIZE DISTRIBUTION MATTERS}

Using a simple metapopulation model (i.e., individuals belong to different populations connected by dispersal), we have shown that disruptive selection on dispersal can occur in natural populations under a larger range of conditions than suggested by previous work (Holt and McPeek 1996; Doebeli and Ruxton 1997; Mathias et al. 2001; Parvinen 2002). Disruptive selection occurs when the distribution of carrying capacity across metapopulation patches is sufficiently skewed, or in simpler terms when the metapopulation is made of few large and many small populations. The reason for this is that a positive skewness of the distribution of carrying capacity ensures that inclusive fitness is variable enough among individuals living in different patch types. Contrary to previous models on the occurrence of disruptive selection on dispersal (McPeek and Holt 1992; Holt and McPeek 1996; Doebeli and Ruxton 1997; Mathias et al. 2001; Parvinen 2002), our model does not assume that local populations are subject to random extinctions, complex population dynamics, or heterogeneity in perturbation regimes.

Parvinen (2002) also predicted that disruptive selection on dispersal can occur in a metapopulation consisting of different patch types. Parvinen (2002) specifically studied the conditions in which disruptive selection on dispersal could occur in a model that allowed for heterogeneous perturbations, carrying capacities, and maximal growth rates. However, in the same vein as Hastings (1983) and Holt (1985), Parvinen (2002) ignored kin competition effects but incorporated indirect dispersal costs due to average migration flows, so that dispersal was selected by perturbations (as in Comins et al. 1980) and counter-selected by heterogeneity in carrying capacities (as in Hastings 1983). Parvinen (2002) found that disruptive selection could occur in metapopulations with heterogeneous perturbation rates (Parvinen 2002, Fig. 4B therein) or heterogeneous carrying capacities (Parvinen 2002, Fig. 5B therein). In the light of inequality (6), our results and Parvinen's share the same rationale, that is, that disruptive selection on dispersal occurs when the selection gradient on dispersal is sufficiently variable among individuals in the metapopulation. In our study, this variability stems from the heterogeneity in kin competition and indirect dispersal costs; in Parvinen (2002), this variability stems from the heterogeneity in perturbation rates and indirect dispersal costs.

Our analytical approach is confirmed by simulations, both with and without demographic stochasticity. In the simplest case, two strategies (drifters and dwellers) can coexist after evolutionary branching: dwellers stably inhabit the few large patches whereas drifters occupy most of the small patches. Dwellers persist in large patches because the cost of dispersal favors the recruitment of residents. Conversely, drifters persist in small patches because dwellers rarely reach them and, when they succeed, do not often disperse to other patches, thus yielding little reward in terms of new colonizations. It should be noted, however, that stabilizing selection does not preclude dispersal polymorphism: it is possible that a dimorphic coalition (with dwellers and drifters) is evolutionarily stable and yet not evolutionarily attainable because selection is stabilizing in monomorphic metapopulations. 


\section{SPATIAL VARIABILITY DOES NOT PREVENT SELECTION FOR DISPERSAL}

In our model, the only factors driving dispersal evolution are the cost of dispersal and spatially heterogeneous patch size, which generates both variation in the intensity of kin competition (kin competition is more intense in small patches) and asymmetry in migrant flow (most migrants leave large patches to enter small patches). Our conclusions apparently contradict those reported on the basis of an earlier model (Hastings 1983; see also Holt 1985) that "spatial variation alone cannot lead to selection for dispersal." However, in that model, patches are described using mean-field dynamics, an approximation that removes stochasticity in the colonization process and effectively suppresses kin competition, the main force selecting for dispersal (Hamilton and May 1977; Cadet et al. 2003). Thus, we intuitively expect Hastings' model to select against dispersal because it does not incorporate kin competition, as predicted by our model under a stabilizing selection regime (eq. 3) when patch size variance is not 0 and the average patch size tends toward infinity (effectively suppressing kin competition effects by lowering relatedness among patch mates). Even in the absence of kin competition, selection for nonzero dispersal is still possible, provided another selective pressure, such as perturbations (Comins et al. 1980) or demographic stochasticity (Cadet et al. 2003), is present.

\section{NATURAL METAPOPULATIONS HAVE SKEWED PATCH SIZE DISTRIBUTIONS}

Are conditions favoring disruptive selection on dispersal frequent in the real world? In five of the seven datasets studied here (see Table 1), our criterion suggests the possibility of disruptive selection. This finding certainly opens the way to empirical evaluation in natural situations in which species have more or less patchy distributions. An encouraging, recurrent observation is that distributions of animal group-size are almost always highly positively skewed (e.g., Thompson and Lyons 1964; Jarman 1974; Wirtz and Lörscher 1983). Aggregation of individual plants (e.g., due to small-tail dispersal kernels and/or to patches of favorable conditions) may also lead to skewed distributions of plant density at low spatial scales (Pélissier and Goreaud 2001).

It is interesting to note that a stable genetic polymorphism of the glycolytic enzyme phosphoglucose isomerase (Pgi), a gene affecting insect mobility and, thus, dispersal ability (Haag et al. 2005), has been observed in natural populations of the Glanville fritillary butterfly M. cinxia on Åland islands (Hanski and Saccheri 2006). This shows that dwellers and drifters can stably coexist in the same species, as predicted by our model. Moreover, $P g i$ polymorphism is not a conspicuous dispersal polymorphism, unlike e.g., wing size in crickets (Roff 1986), variation in the proportion of winged offspring in aphids (Hazell et al. 2005), or in the proportion of seeds with pappus in the Aster- aceae Crepis sancta (Imbert et al. 1997). The findings of Hanski and Saccheri (2006) suggest that inconspicuous dispersal polymorphisms exist; our model proposes that such polymorphisms can be found in metapopulations harboring few large and many small patches.

It should be noted that patch area is taken as a proxy to carrying capacity in five of seven datasets. This is not in complete accordance with our model because we assumed that immigrant pressure is independent of local carrying capacity: under a propagule rain model, it is expected that immigrant pressure is proportional to patch area. Thus, if patch area is taken as a direct proxy for carrying capacity, immigrant pressure is expected to be proportional to carrying capacity (and thus disruptive selection would be impossible because inclusive fitness would be less variable among patch types). Two observations seem to mitigate this:

- propagule rain is unlikely in organisms that disperse actively. In active dispersers, indeed, immigrant pressure is more likely to be linked to the contour of patches (i.e., perimeter in two dimensions, or surface in three dimensions). This would put the "more realistic model" halfway between ours and a model with immigration proportional to carrying capacity;

- Connor et al. (2000) have shown that population density tends on average to increase with area within species. This observation suggests that the skewness and coefficient of variation of carrying capacity assessed through area are underestimated. Thus, using patch areas as proxies of carrying capacities is a conservative option. Moreover, even if immigration is proportional to area (propagule rain assumption), this observation means that immigration will be less than proportional to carrying capacity. These two observations mean that the conclusions stemming from Table 1, although quantitatively imprecise, are qualitatively supported: population sizes are likely to be positively skewed in many cases in nature implying that disruptive selection on dispersal is to be expected.

\section{A TESTABLE THEORY FOR DISPERSAL POLYMORPHISMS}

The creation and maintenance of dispersal polymorphisms is usually explained by the interplay of the selective forces that classically determine dispersal evolution: perturbations, inbreeding depression, dispersal cost, kin competition, habitat heterogeneity, complex population dynamics, and/or environmental fluctuations (Roff 1975; McPeek and Holt 1992; Holt and McPeek 1996; Mathias et al. 2001; Parvinen 2002; Ronce 2007). Our model, by comparison, explains dispersal polymorphisms based only on the existence of dispersal cost and variability in kin competition 
intensity (through variability in carrying capacity). We do not question the existence of perturbations, chaotic population dynamics, or inbreeding depression in natural systems. Moreover, we acknowledge that these factors may have an important influence on dispersal evolution. However, we propose that variability in carrying capacity among patches may often be the factor that triggers disruptive selection on dispersal when all other factors would predict the existence of a unique ESS. Our proposition can be falsified using minimal information on studied metapopulations, contrary to all other hypotheses explaining dispersal polymorphisms. Thus, before invoking perturbations or chaotic population dynamics, the distribution of patch sizes has to be tested using our criterion to assess whether variability in kin competition intensity alone can explain an observed natural polymorphism of dispersal (such as in $M$. cinxia on Åland islands). In general, testing alternative hypotheses is more difficult because it requires data on the spatio-temporal variability of patch sizes or population numbers (Holt and McPeek 1996; Mathias et al. 2001) or on the correlation between patch size and perturbation regime (Parvinen 2002).

\section{DISPERSAL POLYMORPHISM OR PLASTIC DISPERSAL STRATEGY?}

Predicting disruptive selection on dispersal rate does not automatically imply the existence of a genetic polymorphism at loci controlling dispersal abilities, as in the Glanville fritillary butterfly (Hanski and Saccheri 2006). Indeed, circumstances favoring the evolution of multiple fixed dispersal strategies may be equally favorable to the evolution of a complex plastic dispersal strategy (Kisdi 2004; Leimar 2005; Clobert et al. 2009; Enfjall and Leimar 2009). Numerous empirical examples have shown that plasticity of dispersal-related traits is common in nature (Clobert et al. 2001, 2009). However, theoretical investigation of conditiondependent dispersal has begun only recently (Travis et al. 1999; Metz and Gyllenberg 2001; Poethke and Hovestadt 2002; Kisdi 2004; Barton et al. 2009; Enfjall and Leimar 2009), and theories linking results obtained on the evolution of fixed dispersal strategies to condition-dependent dispersal predictions are lacking. If we allow for plastic dispersal strategies in our model, the singular dispersal strategy $d(K)$ is found through nullifying the selection gradient for each value of $K$, that is, $d(K)=\operatorname{Min}[1 /(c K), 1]$ (Appendix S4). Thus, variability in carrying capacities directly fosters plasticity in dispersal ability. Of course, this result assumes that plasticity is not costly and that $K$ can be perceived without error by individuals. In the more likely case of a positive cost to plasticity and limited perceptive ability, we speculate that a highly positively skewed carrying capacity distribution still selects for a decreasing reaction norm (i.e., dispersal decreases with increasing $K$ ).

\section{CONSEQUENCES FOR REALISTIC COMMUNITY MODELS}

Although our model describes the evolution of types in a species living in a metapopulation, it might also be helpful for understanding and enhancing metacommunity models, that is, when considering that each type corresponds to a different species. Because conditions for the evolutionary branching of dispersal rates appear to be quite frequent in nature, we may question the realism of species coexistence models that assume a fixed uniform value for the dispersal rate of all species, such as neutral community models (Hubbell 2001). A consequence of incorporating variability in dispersal abilities among species is that species interactions become nonneutral (grossly, dwellers win in large patches, drifters win in small patches), and thus frequency-dependent selection occurs. Frequency-dependent selection radically changes population dynamics: when a species is rare in the metacommunity, it deterministically promotes either its recovery or its demise. By contrast, in neutral community models, the fate of a rare species is completely random. Even though the initial formulation of our model is "neutral" in the sense that there is no ecological difference between species and there is a single initial dispersal strategy, the addition of evolutionary forces leads to nonneutral dynamics whereby stochastic drift becomes less important. Accounting for the effects of evolution might be the next challenge that community models will have to face to explain current species diversity in ecosystems (Urban and Skelly 2006).

\section{A NEW PERSPECTIVE ON BIOLOGICAL CONSERVATION IN FRAGMENTED LANDSCAPES}

Our results also have important consequences for biodiversity conservation policies. Whether natural reserves should consist of a "Single Large Or Several Small" areas (the so-called SLOSS debate [Hanski and Gilpin 1997]) used to be held as central to the preservation of individual species (but see Semlitsch and Bodie 1998). That communities are often nested (i.e., very rare species only occur in species-rich communities) led to suggesting discarding small reserve patches in favor of fewer large ones (Patterson and Atmar 1986). However, to preserve species diversity within communities and future evolutionary potential (Forest et al. 2007), one should rather foster conditions under which many strategies can emerge and coexist, for example, by increasing habitat diversity to preserve species with different ecological requirements and by maintaining a sufficient amount of hospitable habitats to maintain a balance between fugitive and competitive species (Nee and May 1992). Moreover, a metapopulation hosting types with various dispersal strategies is less likely to collapse after a habitat fragmentation event than a monomorphic metapopulation (Leimar and Norberg 1997). Our model suggests that the conservation of species with different dispersal strategies depends on the spatial 
configuration of the protected habitats, and thus does not depend on an optimum reserve size, but rather on the existence of "Few Large And Many Small” patches.

\section{ACKNOWLEDGMENTS}

The authors thank J. C. Brock, I. Hanski, R. Morteo, and M. PalaseanuLovejoy for access to data, N. Barton, J. Chave, M. Daufresne, S. Gandon, J. Hermisson, M. Leibold, I. Olivieri, O. Ronce, and three anonymous reviewers for comments on the manuscript, J. Auld, V. Calcagno, S. Gandon and N. Mouquet for discussions and advice, M. Daufresne for help with simulations. This work was funded by the French Ministry of Agriculture, Cemagref and EU Marie Curie Action (project DEFTERPLANKTON, contract 236712) funds to FM and by the French Ministry of Agriculture to FM and by CNRS funds to PJ and PD. AD was supported by a doctoral thesis grant from the French Ministry of Research.

\section{LITERATURE CITED}

Ajar, E. 2003. Analysis of disruptive selection in subdivided populations. BMC Evol. Biol. 3:22, doi:10.1186/1471-2148-3-22.

Balkau, B. J., and M. W. Feldman. 1973. Selection for migration modification. Genetics 74:171-174.

Barton, K. A., B. L. Phillips, J. M. Morales, and J. M. J. Travis. 2009. The evolution of an 'intelligent' dispersal strategy: biased, correlated random walks in patchy landscapes. Oikos 118:309-319.

Bengtsson, B. O. 1978. Avoiding inbreeding: at what cost? J. Theor. Biol. 73:439-444.

Brock, J. C., M. Palaseanu-Lovejoy, C. W. Wright, and A. Nayegandhi. 2008. Patch-reef morphology as a proxy for Holocene sea-level variability, Northern Florida Keys, USA. Coral Reefs 27:555-568.

Cadet, C., R. Ferriere, J. A. J. Metz, and M. van Baalen. 2003. The evolution of dispersal under demographic stochasticity. Am. Nat. 162:427-441.

Champagnat, N., R. Ferriere, and S. Meleard. 2006. Unifying evolutionary dynamics: from individual stochastic processes to macroscopic models. Theor. Popul. Biol. 69:297-321.

Charlesworth, B. 1994. Evolution in age-structured populations, 2nd ed. Cambridge Univ. Press, Cambridge.

Chesson, P. L. 1984. Persistence of a Markovian population in a patchy environment. Z Wahrscheinlichkeit. 66:97-107.

Clobert, J., E. Danchin, A. A. Dhondt, and J. D. Nichols. 2001. Dispersal. Oxford Univ. Press, New York.

Clobert, J., J.-F. Le Galliard, J. Cote, S. Meylan, and M. Massot. 2009. Informed dispersal, heterogeneity in animal dispersal syndromes and the dynamics of spatially structured populations. Ecol. Lett. 12:197-209.

Comins, H. N., W. D. Hamilton, and R. M. May. 1980. Evolutionarily stable dispersal strategies. J. Theor. Biol. 82:205-230.

Connor, E. F., A. C. Courtney, and J. M. Yoder. 2000. Individuals-area relationships: the relationship between animal population density and area. Ecology 81:734-748.

Doebeli, M., and G. D. Ruxton. 1997. Evolution of dispersal rates in metapopulation models: branching and cyclic dynamics in phenotype space. Evolution 51:1730-1741.

Enfjall, K., and O. Leimar. 2009. The evolution of dispersal-the importance of information about population density and habitat characteristics. Oikos 118:291-299.

Forest, F., R. Grenyer, M. Rouget, T. J. Davies, R. M. Cowling, D. P. Faith, A. Balmford, J. C. Manning, S. Proches, M. Van Der Bank, et al. 2007. Preserving the evolutionary potential of floras in biodiversity hotspots. Nature 445:757-760.

Gandon, S., and Y. Michalakis. 2001. Multiple causes for the evolution of dispersal. Pp. 155-167 in J. Clobert, E. Danchin, A. A. Dhondt and J. D. Nichols, eds. Dispersal. Oxford Univ. Press, New York.

Geritz, S. A. H., E. Kisdi, G. Meszena, and J. A. J. Metz. 1998. Evolutionarily singular strategies and the adaptive growth and branching of the evolutionary tree. Evol. Ecol. 12:35-57.

Haag, C. R., M. Saastamoinen, J. H. Marden, and I. Hanski. 2005. A candidate locus for variation in dispersal rate in a butterfly metapopulation. Proc. R. Soc. Biol. Lond. B 272:2449-2456.

Hamilton, W. D., and R. M. May. 1977. Dispersal in stable habitats. Nature 269:578-581.

Hanski, I., and M. E. Gilpin. 1997. Metapopulation biology: ecology, genetics, and evolution. Academic Press, San Diego, CA.

Hanski, I., and I. Saccheri. 2006. Molecular-level variation affects population growth in a butterfly metapopulation. PLoS Biol. 4:719-726.

Hastings, A. 1983. Can spatial variation alone lead to selection for dispersal? Theor. Popul. Biol. 24:244-251.

Hazell, S. P., D. M. Gwynn, S. Ceccarelli, and M. D. E. Fellowes. 2005. Competition and dispersal in the pea aphid: clonal variation and correlations across traits. Ecol. Entomol. 30:293-298.

Hofbauer, J., and K. Sigmund. 1990. Adaptive dynamics and evolutionary stability. Appl. Math. Lett. 3:75-79.

Holt, R. D. 1985. Population dynamics in two-patch environments: some anomalous consequences of an optimal habitat distribution. Theor. Popul. Biol. 28:181-208.

Holt, R. D., and M. A. McPeek. 1996. Chaotic population dynamics favors the evolution of dispersal. Am. Nat. 148:709-718.

Hubbell, S. P. 2001. The unified neutral theory of biodiversity and biogeography. Princeton Univ. Press, Princeton, NJ.

Imbert, E., J. Escarré, and J. Lepart. 1997. Seed heterodimorphism in Crepis sancta (Asteraceae): performance of two morphs in different environments. Oikos 79:325-332.

Jansen, V. A. A., and R. Vitalis. 2007. The evolution of dispersal in a Levins' type metapopulation model. Evolution 61:2386-2397.

Jarman, P. J. 1974. The social organisation of antelope in relation to their ecology. Behaviour 48:215-267.

Kisdi, E. 2002. Dispersal: risk spreading versus local adaptation. Am. Nat. 159:579-596.

Kisdi, E. 2004. Conditional dispersal under kin competition: extension of the Hamilton-May model to brood size-dependent dispersal. Theor. Popul. Biol. 66:369-380.

Leimar, O. 2005. The evolution of phenotypic polymorphism: randomized strategies versus evolutionary branching. Am. Nat. 165:669-681.

Leimar, O., and U. Norberg. 1997. Metapopulation extinction and genetic variation in dispersal-related traits. Oikos 80:448-458.

Levin, S. A., H. C. Muller-Landau, R. Nathan, and J. Chave. 2003. The ecology and evolution of seed dispersal: a theoretical perspective. Annu. Rev. Ecol. Syst. 34:575-604.

Massol, F., V. Calcagno, and J. Massol. 2009. The metapopulation fitness criterion: proof and perspectives. Theor. Popul. Biol. 75:183-200.

Mathias, A., E. Kisdi, and I. Olivieri. 2001. Divergent evolution of dispersal in a heterogeneous landscape. Evolution 55:246-259.

McPeek, M. A., and R. D. Holt. 1992. The evolution of dispersal in spatially and temporally varying environments. Am. Nat. 140:1010-1027.

Metz, J. A. J., and M. Gyllenberg. 2001. How should we define fitness in structured metapopulation models? Including an application to the calculation of evolutionarily stable dispersal strategies. Proc. R. Soc. Biol. Sci. Lond. B 268:499-508.

Nee, S., and R. M. May. 1992. Dynamics of metapopulations: habitat destruction and competitive coexistence. J. Anim. Ecol. 61:37-40.

Parvinen, K. 2002. Evolutionary branching of dispersal strategies in structured metapopulations. J. Math. Biol. 45:106-124. 
Patterson, B. D., and W. Atmar. 1986. Nested subsets and the structure of insular mammalian faunas and archipelagos. Biol. J. Linn. Soc. 28:6582.

Pélissier, R., and F. Goreaud. 2001. A practical approach to the study of spatial structure in simple cases of heterogeneous vegetation. J. Veg. Sci. 12:99-108.

Poethke, H. J., and T. Hovestadt. 2002. Evolution of density- and patch-sizedependent dispersal rates. Proc. R. Soc. Biol. Sci. Lond. B 269:637-645.

Primack, R. B., and R. T. Corlett. 2005. Tropical rain forests: an ecological and biogeographical comparison. Blackwell Publishing, Oxford, UK.

Roff, D. A. 1975. Population stability and the evolution of dispersal in a heterogeneous environment. Oecologia 19:217-237.

Roff, D. A. 1986. The evolution of wing dimorphisms in insects. Evolution 40:1009-1020.

Ronce, O. 2007. How does it feel to be like a rolling stone? Ten questions about dispersal evolution. Annu. Rev. Ecol. Evol. Syst. 38:231-253.

Ronce, O., F. Perret, and I. Olivieri. 2000. Evolutionarily stable dispersal rates do not always increase with local extinction rates. Am. Nat. 155:485496.
Roze, D., and F. Rousset. 2005. Inbreeding depression and the evolution of dispersal rates: a multilocus model. Am. Nat. 166:708-721.

Semlitsch, R. D., and J. R. Bodie. 1998. Are small, isolated wetlands expendable? Conserv. Biol. 12:1129-1133.

Thompson, D. Q., and M. D. Lyons. 1964. Flock size in a spring concentration of Whistling Swans. Wilson Bull. 76:282-285.

Travis, J. M. J., D. J. Murrell, and C. Dytham. 1999. The evolution of density-dependent dispersal. Proc. R. Soc. Biol. Sci. Lond. B 266:18371842.

Urban, M. C., and D. K. Skelly. 2006. Evolving metacommunities: toward an evolutionary perspective on metacommunities. Ecology 87:16161626.

van Valen, L. 1971. Group selection and the evolution of dispersal. Evolution 25:591-598.

Wirtz, P., and J. Lörscher. 1983. Group sizes of Antelopes in an East African national park. Behaviour 84:135-156.

Wood, R. 2001. Biodiversity and the history of reefs. Geol. J 36:251-263.

Associate Editor: J. Hermisson

\section{Supporting Information}

The following supporting information is available for this article:

Appendix S1. Complete mathematical analysis of the model.

Appendix S2. Application of the model to geometric distributions.

Appendix S3. Accounting for demographic stochasticity.

Appendix S4. Computing the plastic ESS.

Figure S1. Simulation results for $p=0.2$ (example of stabilizing selection on dispersal).

Figure S2. Simulation results for $p=0.5$ (example of disruptive selection on dispersal).

Figure S3. Simulation results for $p=0.5$ with demographic stochasticity.

Supporting Information may be found in the online version of this article.

Please note: Wiley-Blackwell is not responsible for the content or functionality of any supporting information supplied by the authors. Any queries (other than missing material) should be directed to the corresponding author for the article. 